\title{
Landscape structure shapes carnivore-mediated seed dispersal kernels
}

\author{
José M. Herrera $\cdot$ Isa de Sá Teixeira $•$ \\ Javier Rodríguez-Pérez • \\ António Mira
}

Received: 17 April 2015/Accepted: 21 September 2015/Published online: 28 September 2015

(c) Springer Science+Business Media Dordrecht 2015

\begin{abstract}
Context Seed dispersal is recognized as having profound effects on the distribution, dynamics and structure of plant populations and communities. However, knowledge of how landscape structure shapes carnivore-mediated seed dispersal patterns is still scarce, thereby limiting our understanding of large-scale plant population processes.

Objectives We aim to determine how the amount and spatial configuration of forest cover impacted the relative abundance of carnivorous mammals, and how these effects cascaded through the seed dispersal kernels they generated.

Methods Camera traps activated by animal movement were used for carnivore sampling. Colour-coded seed mimics embedded in common figs were used to know the exact origin of the dispersed seed mimics
\end{abstract}

Electronic supplementary material The online version of this article (doi:10.1007/s10980-015-0283-4) contains supplementary material, which is available to authorized users.

J. M. Herrera $(\bowtie) \cdot$ A. Mira

Research Center in Biodiversity and Genetic Resources University of Évora (CIBIO/InBIO-UE), 7000-890 Évora, Portugal

e-mail: herreramirlo@gmail.com

Present Address:

J. M. Herrera

Estación Biológica de Doñana (EBD-CSIC), Avd.

Américo Vespucio s/n, Isla de la Cartuja, 41092 Seville, Spain later found in carnivore scats. We applied this procedure in two sites differing in landscape structure. Results We did not find between-site differences in the relative abundance of the principal carnivore species contributing to seed dispersal patterns, Martes foina. Mean dispersal distance and the probability of long dispersal events were higher in the site with spatially continuous and abundant forest cover, compared to the site with spatially aggregated and scarcer forest cover. Seed deposition closely matched the spatial patterning of forest cover in both study sites, suggesting behaviour-based mechanisms underpinning seed dispersal patterns generated by individual frugivore species.

Conclusions Our results provide the first empirical evidence of the impact of landscape structure on carnivore-mediated seed dispersal kernels. They also indicate that seed dispersal kernels generated strongly depend on the effect that landscape structure exerts on carnivore populations, particularly on habitat-use preferences.

I. de Sá Teixeira

Deptartment Animal Biology, University of Lisbon, 1749-016 Lisbon, Portugal

J. Rodríguez-Pérez

Deptartment Ornithology, Aranzadi Sciences Society, Zorroagagaina 11, 20014 Donostia-San Sebastián, Spain

A. Mira

Conservation Biology Unit, Deptartment of Biology, University of Évora (UBC-UE), 7002-554 Évora, Portugal 